\title{
The assessment of skeletal status in young patients with Turner syndrome by 2 densitometric techniques: Phalangeal quantitative ultrasound and dual energy X-ray absorptiometry
}

\author{
Beata Wikiera ${ }^{1, A-D, F}$, Agata Mierzwicka ${ }^{2, B, C}$, Aleksander Basiak ${ }^{1, A-C}$, Jowita Halupczok-Żyła ${ }^{2, B, C}$, \\ Diana Jędrzejuk ${ }^{2, A-C}$, Magdalena Cabała ${ }^{3, B}$, Anna Noczyńska ${ }^{1, A, E}$, Marek Bolanowski ${ }^{2, A, E, F}$, \\ Kornel Mikołajczyk $k^{4, B, E}$, Zenon P. Halaba ${ }^{5, A-F}$ \\ 1 Department and Clinic of Endocrinology and Diabetology for Children and Adolescents, Wroclaw Medical University, Poland \\ 2 Department and Clinic of Endocrinology, Diabetes and Isotope Therapy, Wroclaw Medical University, Poland \\ ${ }^{3}$ Department of Pediatrics, Division of Propedeutics of Pediatrics and Rare Disorders, Wroclaw Medical University, Poland \\ ${ }^{4}$ Faculty of Rehabilitation, The Józef Piłsudski University of Physical Education in Warsaw, Poland \\ ${ }^{5}$ Department of Medical Simulation, University of Opole, Poland \\ A - research concept and design; $B$ - collection and/or assembly of data; $C$ - data analysis and interpretation; \\ $\mathrm{D}$ - writing the article; $\mathrm{E}$ - critical revision of the article; $\mathrm{F}$ - final approval of the article
}

Address for correspondence

Zenon P. Halaba

E-mail:zhalaba@uni.opole.pl

\section{Funding sources}

None declared

Conflict of interest

None declared

\section{Acknowledgements}

We thank Prof. Laura K. Bachrach from Stanford University for her assistance in calculating the height-adjusted Z-score based on the Z-score calculator of the Bone Mineral Density in Childhood Study (BMDCS) for Hologic systems.

Received on December 18, 2016 Reviewed on February 26, 2017 Accepted on June 8, 2017

DOI

10.17219/acem/74598

Copyright

Copyright by Author(s)

This is an article distributed under the terms of the

Creative Commons Attribution Non-Commercial License

(http://creativecommons.org/licenses/by-nc-nd/4.0/)

\begin{abstract}
Background. Studies using dual energy X-ray absorptiometry (DXA) demonstrate a reduction in bone mineral density (BMD) in children and adolescents with Turner syndrome (TS). However, these studies do not take into account changes in bone size, which influence BMD in the case of short-statured patients. Phalangeal quantitative ultrasound (phQUS) measurements have shown an ability to reveal changes due to skeletal growth, aging, and bone and mineral disorders. There is limited data on bone mineral status in girls with TS assessed by 2 different techniques, i.e., DXA and phQUS.
\end{abstract}

Objectives. The aim of this study was to investigate the potential negative impact of TS on bone status and to assess whether densitometric values were related to former fractures.

Material and methods. In 43 TS girls aged 5-18 years, we evaluated bone status by 2 different densitometric techniques, DXA and phQUS.

Results. The mean lumbar spine areal bone mineral density (LS aBMD) Z-score was significantly lower than 0 (the hypothetical mean) compared to the reference population $(p<0.001)$. The mean LS aBMD height-adjusted Z-score did not differ significantly from 0 . The amplitude-dependent speed of sound (Ad-SoS) Z-score was significantly lower than 0 compared with a Polish reference population. There were no significant differences between fractured and fracture-free patients as regards Ad-SOS Z-score and LS aBMD height-adjusted Z-score.

Conclusions. Girls with TS have normal bone density adjusted for height, but significantly decreased phQUS values. Neither DXA nor phalangeal Ad-SoS can identify young TS patients with former fractures.

Key words: fractures, quantitative ultrasound, Turner syndrome, dual energy X-ray absorptiometry 


\section{Introduction}

An increased risk of fracture is a feature of Turner syndrome (TS). However, the reason for this is unclear, and forearm fractures have been estimated to be more prevalent in the TS population. ${ }^{1}$ Some studies also reported an increase in fracture prevalence during childhood in these patients. ${ }^{2}$ Exogenous recombinant human growth hormone (rhGH) is often used in childhood to increase height. The role of growth hormone (GH) treatment in promoting bone accrual has not yet been completely defined, although the administration of $\mathrm{GH}$ is known to increase bone mineral density (BMD) in children without TS. ${ }^{3,4}$ Studies using dual energy X-ray absorptiometry (DXA) demonstrate a reduction in BMD in children and adolescents with TS. ${ }^{5-7}$ However, these studies do not take into account changes in bone size, which influence BMD in the case of short-statured patients. DXA studies, when adjusted for bone size, find no differences in volumetric BMD (vBMD) between TS girls and controls. ${ }^{8}$ Peripheral quantitative computed tomography (pQCT) is able to provide precise measurements of 3-dimensional bone density without being influenced by bone size. In TS girls, pQCT data suggests that cortical density is reduced with sparing of trabecular bone. ${ }^{9}$ In recent years, another promising method of assessing skeletal status was introduced. This method is based on ultrasound waves, which are transmitted through the bone tissue. Phalangeal quantitative ultrasound (phQUS) measurements have demonstrated an ability to reveal changes due to skeletal growth, aging, and bone and mineral disorders. ${ }^{10-14}$ Quantitative ultrasound (QUS) is also devoid of ionizing radiation, and is cost-effective, easy to use and portable - all highly desirable features in pediatrics. There is data on girls with TS whose bone mineral status was assessed by 2 different techniques, i.e., DXA and phQUS. Despite its pitfalls, DXA is recommended as a monitoring tool in children with chronic disease who are at risk of developing osteoporosis; phQUS, however, is able to provide measurements of bone quality without being influenced by bone size. The objective of our study was to assess bone status in young girls with TS by 2 different densitometric techniques, DXA and phQUS, and to evaluate the relationship between low bone density and the prevalence of fracture.

This is the first study to concurrently examine the lumbar spine (LS) BMD height-adjusted Z-score and amplitude-dependent speed of sound (Ad-SoS) Z-score in Polish TS girls.

\section{Material and methods}

\section{Patients}

In the study, 43 Caucasian Polish girls with TS aged $13.7 \pm 3.4$ years (range 5.3-18.3) were enrolled as outpatients to the Department and Clinic of Endocrinology and
Table 1. Clinical findings in TS girls

\begin{tabular}{|c|c|c|}
\hline Variable & $\begin{array}{c}\text { Mean } \pm S D \\
\text { (range) }\end{array}$ & $p$-value \\
\hline Age [years] & $\begin{array}{l}13.7 \pm 3.4 \\
(5.3-18.3)\end{array}$ & NA \\
\hline Height SDS & $\begin{array}{l}-1.9 \pm 1.1 \\
(-5.4-0.3)\end{array}$ & $0.001^{*}$ \\
\hline Weight SDS & $\begin{array}{l}-0.26 \pm 1.3 \\
(-2.0-3.6)\end{array}$ & $\mathrm{NS}^{*}$ \\
\hline BMI SDS & $\begin{array}{c}1.1 \pm 1.7 \\
(-1.5-4.8)\end{array}$ & $0.01^{*}$ \\
\hline Age at diagnosis [years] & $\begin{array}{c}6.1 \pm 4.9 \\
(0.1-15.1)\end{array}$ & NA \\
\hline Age at start of rhGH therapy [years] & $\begin{array}{c}8.1 \pm 3.9 \\
(3.6-14.3)\end{array}$ & NA \\
\hline Cumulative dose of rhGH [mg] & $2375 \pm 1802$ & NA \\
\hline Ad-SoS Z-score & $\begin{array}{l}-1.1 \pm 1.26 \\
(-3.9-1.8)\end{array}$ & $0.001^{*}$ \\
\hline LS aBMD Z-score & $\begin{array}{c}-0.84 \pm 1.04 \\
(-3.9-0.9)\end{array}$ & $0.001^{*}$ \\
\hline LS aBMD height-adjusted Z-score & $\begin{array}{l}-0.21 \pm 1.1 \\
(-3.7-1.6)\end{array}$ & $\mathrm{NS}^{*}$ \\
\hline
\end{tabular}

* comparison of SDS axiological and Z-score densitometric variables with the hypothetical mean of 0.0; SDS - standard deviation score; BMI - body mass index; rhGH - recombinant human growth hormone; Ad-SoS - amplitude-dependent speed of sound; LS aBMD - lumbar spine areal bone mineral density; NS - not significant; NA - not applicable.

Diabetology for Children and Adolescents or the Department and Clinic of Endocrinology, Diabetes and Isotope Therapy of Wroclaw Medical University, Poland. They were sent an invitation with an informational letter. After consent was given, a questionnaire detailing fracture history was completed by the participants and/or their parents or guardians. The diagnosis of TS was made by karyotyping $(45, X=22$, deletion $=4$ and mosaic $=17)$. The clinical characteristics of the study group are presented in Table 1.

None of our patients suffered from other conditions known to be associated with disrupted mineral metabolism. Recombinant human growth hormone was administered at a dose of $0.33 \mathrm{mg} / \mathrm{kg} /$ week. In all patients diagnosed before puberty, pubertal development was induced at the age of 12 with the most physiological form of estrogen available for replacement. Starting from a daily dose of $0.25 \mathrm{mg}, 17 \beta$-estradiol was administered orally in increasing doses. Progesterone was added after at least 1 year of estrogenization. In case of a late diagnosis of TS, rhGH was administered first, and pubertal induction began 1 year later. Twenty-three girls had been treated with estrogens and only 6 with hormone replacement therapy (HRT).

\section{Study design}

In order to assess skeletal status in all patients, we evaluated LS BMD by DXA, and Ad-SoS using QUS. Phalangeal QUS results were compared with reference data for 
Polish girls and the Ad-SOS Z-score was calculated. ${ }^{13,14}$ From the DXA results - in order to avoid misdiagnosis in the case of the assessment of short individuals - we calculated LS aBMD height-adjusted Z-score based on the Z-score calculator of the Bone Mineral Density in Childhood Study (BMDCS) for Hologic Systems, which was sponsored by the Eunice Kennedy Shriver National Institute of Childhood and Human Development (NICHD). The normative data from this project is currently considered to be the gold standard for pediatric norms. ${ }^{15}$ The LS aBMD height-adjusted Z-score deals better with the stature issue than bone mineral apparent density (BMAD) Z-score. ${ }^{16}$ Standing height was measured with a floor-mounted stadiometer, body weight was measured to the nearest $0.1 \mathrm{~kg}$ with a standard medical scale, and body mass index (BMI) was calculated $\left[\mathrm{kg} / \mathrm{m}^{2}\right]$. Height, weight, and BMI were expressed as a standard deviation score (SDS) and were calculated using references from the Polish general population.

Informed verbal consent was obtained from all girls and written consent was given by parents/guardians and the girls who were older than 18 years. The study was approved by the Ethics Committee at Wroclaw Medical University.

\section{Assessment of bone mineral status and the prevalence of fracture}

Phalangeal QUS was measured using a DBM Sonic Bone Profiler (Igea, Carpi, Modena, Italy), which measures the amplitude-dependent speed of sound $[\mathrm{m} / \mathrm{s}]$ at the proximal phalanges of dominant-hand fingers. Ad-SoS [m/s] was measured in the distal metaphyses of the proximal phalanges of the $2^{\text {nd }}-5^{\text {th }}$ fingers of the dominant hand, taking into account the first signal with an amplitude of at least $2 \mathrm{mV}$ at the receiving probe. Thus, the measured speed of sound is specified as "amplitude-dependent." All phQUS measurements were performed by the same operator (ZPH). The precision error for QUS results was previously established by 5 serial measurements and expressed as the root-mean-square coefficient of variation (RMS-\%CV). RMS-\%CV for Ad-SoS was $0.64 \%$.

Lumbar spine areal bone mineral density (LS aBMD) was measured by a Discovery W densitometer (Hologic Inc., Bedford, USA) using APEX software (release 4.5.2.1, Hologic Inc.), and the LS aBMD Z-score was calculated. The coefficient of precision was about $1 \%$ for LS aBMD.

For all patients, the frequency and severity of injury and the sites of fractures were recorded and classified according to the Landin severity scale. ${ }^{17}$

\section{Statistical analysis}

From the phQUS and DXA results obtained, Z-scores were calculated as the difference between the patient's value and the age-specific mean, divided by the reference group's standard deviation $(\mathrm{SD})[\mathrm{Z}$-score $=$ (test result for patient - age-specific mean in reference population)/agespecific SD in reference population].

All calculations were done using R v. 3.2.4 (R Foundation for Statistical Computing, Vienna, Austria). Descriptive statistics were presented as mean values, standard deviations, and value ranges for continuous variables. Distribution normality was tested by the Shapiro-Wilk test of normality. In the case of nonparametric data, the Yeo-Johnson transformation was used to reduce skewness and to approximate normality. For a comparative analysis of normally distributed variables, the ANOVA algorithm, Student's t-test, and multiple comparisons of means by Tukey contrasts were used. For comparative analysis of non-normal distributed variables, the Fisher-Pitman permutation test was used. Depending on the distribution of the variables analyzed, Pearson's or Spearman's correlation coefficients were used to estimate the associations between 2 variables. Significance was assumed at $\mathrm{p}<0.05$.

\section{Results}

A total of 43 girls with genetically confirmed TS aged 5-18 years participated in the study. The mean height SDS was significantly below that of age-matched Polish girls, whereas the mean weight SDS did not differ significantly from that of age-matched Polish girls, and the mean BMI SDS was significantly greater (Table 1 ). Of the 43 girls, 7 (16\%) were prepubertal, 22 (51\%) had reached Tanner stage 2 or higher pubarche or breast development without menarche, and 14 (33\%) were postmenarche. Although the mean LS aBMD Z-score was significantly lower than 0 (the hypothetical mean) when compared with a reference population ( $\mathrm{p}=0.001$ ), the mean LS aBMD height-adjusted Z-score did not differ significantly from 0 (Fig. 1). The AdSoS Z-score was significantly lower than 0 compared to a Polish reference population ( $\mathrm{p}=0.001$ ) (Fig. 2). The LS aBMD height-adjusted Z-score was $\leq-2$ in 1 out of 43 girls (2\%), and the Ad-SoS Z-score was $\leq-2$ in 11 out of 43 girls (26\%). None of the patients reported pathological fractures. Nine girls had fractures caused by medium-force-trauma (21\%), including 1 patient who reported 2 fractures. All fractures involved the appendicular skeleton, but not vertebral or femoral sites. We did not find significant differences between patients with and without fractures as regards the Ad-SoS and LS aBMD height-adjusted Z-scores. Only 1 girl, who had sustained 2 fractures, had an LS aBMD height-adjusted Z-score of $<-2$; the others showed normal values. The Ad-SoS Z-score was $\leq-2$ in 3 girls with a positive history of fracture (including the girl with 2 fractures). We did not find any differences in Ad-SoS and BMD values in relation to karyotype or age of diagnosis. The DXA and QUS parameters did not correlate with age at the start of GH therapy and cumulative doses of GH. The LS BMD values differed significantly between girls with breast Tanner stage 4 or lower (mean \pm SD: $-0.39 \pm 1.06$ ), and those with stage 5 (mean \pm SD: $0.87 \pm 0.64$ ) (p<0.03). Ad-SoS 


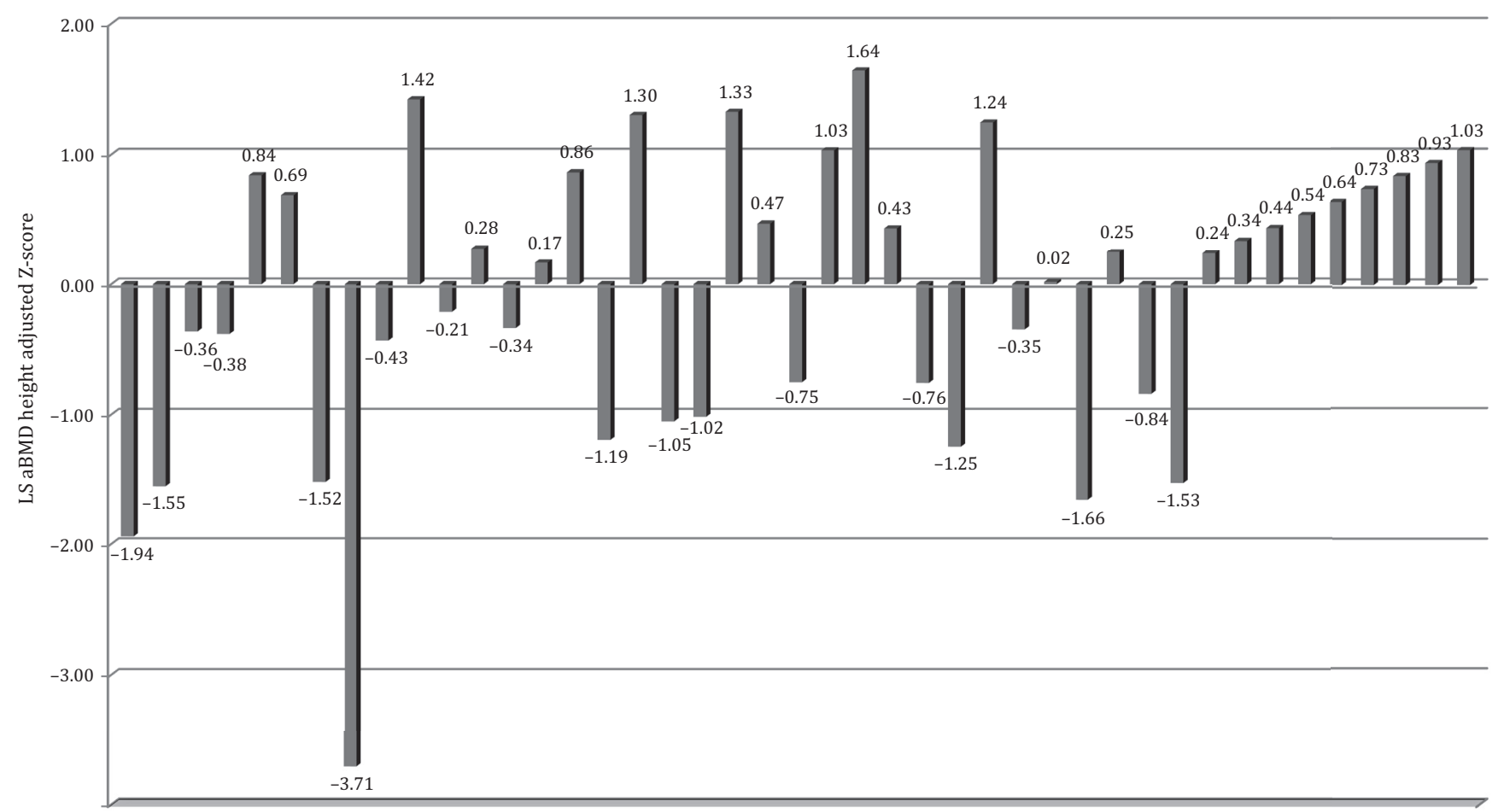

serial number of patients

Fig. 1. Individual values of LS aBMD height-adjusted Z-score

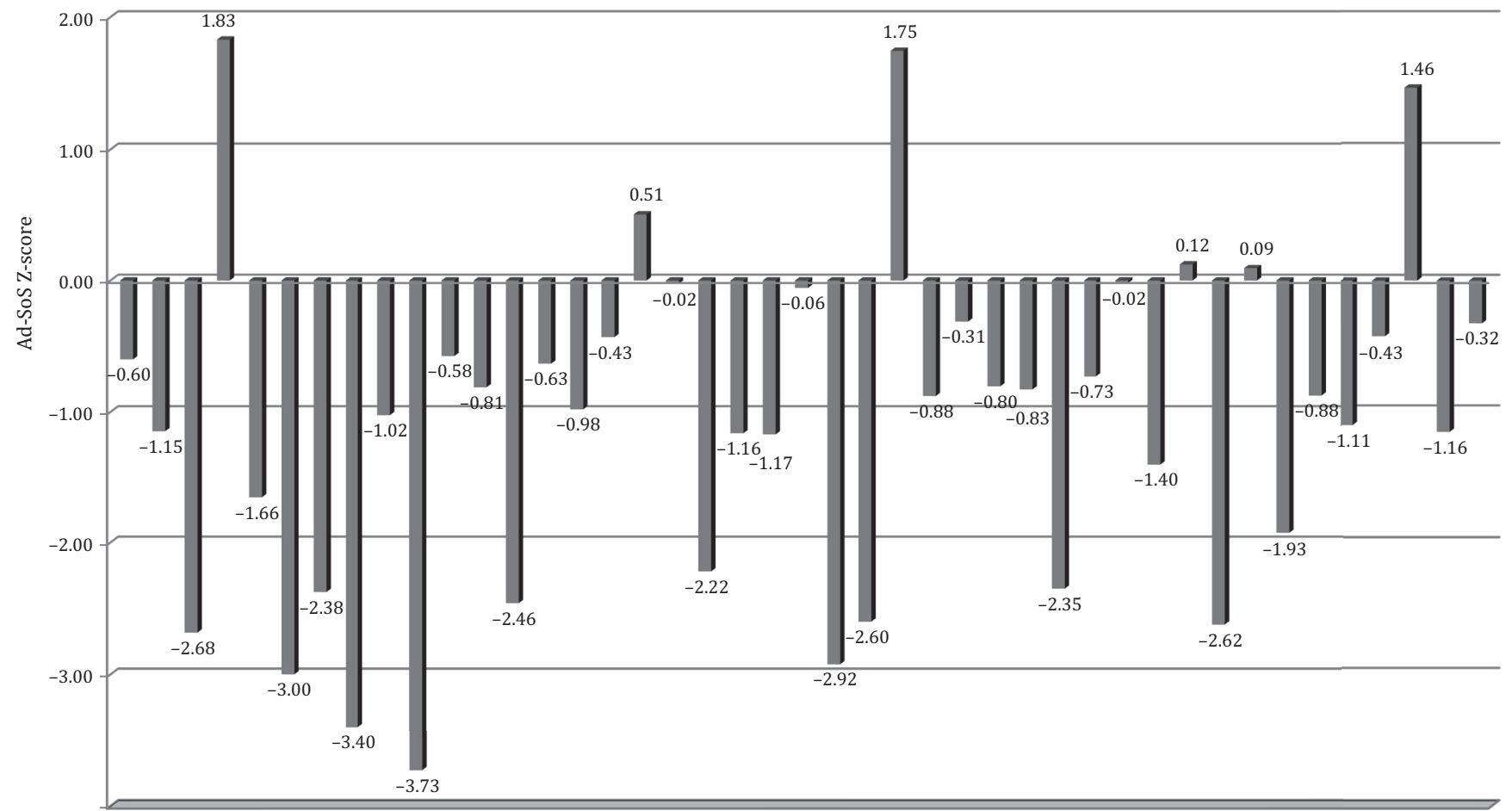

serial number of patients

Fig. 2. Individual values of Ad-SoS Z-score

values - but not DXA values - showed only a significantly negative correlation with the presence of menstrual disturbances $(\mathrm{p}<0.03)$.

\section{Discussion}

Although short stature is the most common clinical feature of TS, skeletal abnormalities encompass more than poor linear growth. One of the biggest concerns is that 
of increased fracture risk and decreased bone density. Studies using DXA show decreased BMD, but small size leads to underestimation, and when the results are adjusted for body size, patients with TS usually have normal BMD in the spine, i.e., in trabecular bone. ${ }^{2,18,19}$ This phenomenon also occurred in our study, showing that apparent BMD may be misleading when evaluating patients with short stature. Although the majority of studies indicate that the abnormalities seem to be only in cortical bone, some studies show compromised trabecular microarchitecture in patients with TS. ${ }^{20,21}$ However, reduced cortical BMD in young TS patients is not proven to lead to increased fractures. ${ }^{22-24}$

We assessed the skeletal status of a group of young females with TS at 2 different skeletal sites using 2 different densitometric techniques. Phalangeal QUS measurements have shown an ability to reveal changes due to skeletal disorders and seem to be less influenced by bone size. ${ }^{10-12}$ Furthermore, as proximal phalanges of the hands consist primarily of cortical bone ( $>60 \%)$, a phalangeal QUS evaluation may provide more information on bone health in TS girls than DXA. Our patients had statistically significantly lower Ad-SoS values compared to healthy Polish girls, but not LS BMD values after adjusting for height. These findings are in agreement with other studies demonstrating that in young TS girls, at a site with predominantly trabecular bone (i.e., the lumbar spine), BMD does not differ from controls after adjusting for body size. , $^{2,18,19,25}$ However, reduced BMD may be present at sites of predominantly cortical bone, such as the $1 / 3$ distal radius. ${ }^{23}$ Also, the studies that used pQCT, a method which is able to independently provide measurements of 3-dimensional bone density and an assessment of trabecular and cortical bone density, have revealed significantly reduced cortical volumetric BMD (vBMD) and cortical thickness at some sites, and lower bone strength. ${ }^{20-22}$ Holroyd et al. showed by this method not only a significant reduction in cortical vBMD at the proximal radius, but in cortical thickness as well (Z-score of -2.58 and -2.89 , respectively). ${ }^{9}$ Moreover, they found no differences in the total vBMD Z-score or the trabecular vBMD Z-score at the distal radius. Using ultrasound methods, comparable results were obtained by Zuckerman-Levin et al. and Vierucci et al. ${ }^{24,26}$ As in our study, they found reduced phalangeal Ad-SoS, and radial and tibial SoS in girls and women with TS. As with pQCT data, abnormal QUS results may imply structural abnormalities in cortical bone in TS. In contrast, Soucek et al. questioned the findings that the cortical BMD is decreased in TS. They concluded that the partial volume effect is probably responsible for this outcome. ${ }^{27}$ The fracture prevalence in our patients was $21 \%$, and was similar to other observations. ${ }^{28,29}$ This percentage is not higher than in a healthy population, because fractures are also common in youth, with $1 / 3$ of all children sustaining at least 1 fracture before the age of 17 years. ${ }^{30,31}$ Our finding varies from a paper by Vierucci et al. ${ }^{26}$ They found a slightly increased risk of fracture in TS girls. In the study,
62.5\% of their TS patients had an Ad-SoS Z-score of $<-2$ and only 1 TS patient with positive history of fracture had normal QUS values, whereas $67 \%$ of our TS girls with a previous history of fracture had an Ad-SoS Z-score of $>-2$. We also did not find any differences in Ad-SoS values between TS girls with and without a positive history of fracture. Unfortunately, we did not assess bone transmission time (BTT), which was shown by Vierucci et al. to have a strong correlation with previous fractures. These differences are difficult to explain. Perhaps this conflicting data resulted from the earlier age of our patients at the start of rhGH therapy, but we found no correlation between QUS values and the onset of GH treatment. Also, the relatively small number of subjects included in these studies may be behind the dissimilarities encountered. Unlike Vierucci et al., we examined the dominant hand, because the Polish reference values were calculated on the basis of dominant hand measurements, ${ }^{14}$ but studies by Shönau et al. and Baroncelli et al. have showen that there is no significant difference between measurements of the two hands. ${ }^{32,33}$ Although GH administration, the standard treatment for TS patients, is considered to promote bone accrual and strength, we found no correlation between the cumulative dose of GH and densitometric results. ${ }^{21}$ Similar results have been obtained by other authors. ${ }^{5,34,35}$ Furthermore, karyotype did not influence densitometric Z-score values, which was also in an agreement with previous studies. ${ }^{34,36}$

Our study may have been limited by its cross-sectional design and the small number of subjects studied. However, the rarity of TS limits the potential for enrollment. Indeed, other studies assessing skeletal status in young TS patients encompass a similar number of subjects. A longitudinal study in patients with TS is in progress, and the results of this study may furnish further important data.

\section{Conclusions}

We concluded that TS girls have normal bone density adjusted for height, but significantly decreased phQUS values. Neither DXA nor phalangeal Ad-SoS can identify young TS patients with former fractures.

\section{References}

1. Gravholt CH, Vestergaard P, Hermann AP, Mosekilde L, Brixen K, Christiansen JS. Increased fracture rates in Turner's syndrome: A nationwide questionnaire survey. Clin Endocrinol. 2003;59:89-96.

2. Ross JL, Long LM, Feuillan P, Cassorla F, Cutler GB Jr. Normal bone density of the wrist and spine and increased wrist fractures in girls with Turner's syndrome. J Clin Endocrinol Metab. 1991;73:355-359.

3. Saggese G, Baroncelli GI, Bertoelloni S, Barsanti S. The effect of longterm growth hormone $(\mathrm{GH})$ treatment on bone mineral density in children with $\mathrm{GH}$ deficiency. Role of GH in the attainment of peak bone mass. J Clin Endocrinol Metab. 1996;81:3077-3083.

4. Conway GS, Szarras-Czapnik M, Racz K, et al. Treatment for 24 months with recombinant human $\mathrm{GH}$ has a beneficial effect on bone mineral density in young adults with childhood-onset GH deficiency. Eur J Endocrinol. 2009;160(6):899-907. 
5. Aycan Z, Cetinkaya E, Darendeliler F, et al. The effect of growth hormone treatment on bone mineral density in prepubertal girls with Turner syndrome: A multicentre prospective clinical trial. Clin Endocrinol (Oxf). 2008;68(5):769-772.

6. Davies MC, Gulekli B, Jacobs HS. Osteoporosis in Turner's syndrome and other forms of primary amenorrhoea. Clin Endocrinol. 1995;43: 741-746.

7. Landin-Wilhelmsen K, Bryman I, Windh M, Wilhelmsen L. Osteoporosis and fractures in Turner syndrome: Importance of growth promoting and oestrogen therapy. Clin Endocrinol. 1999;51:497-502.

8. Nadeem $M$, Roche EF. Bone health in children and adolescent with Turner syndrome. J Pediatr Endocrinol Metab. 2012;25:823-833.

9. Holroyd CR, Davies JH, Taylor P, et al. Reduced cortical bone density with normal trabecular bone density in girls with Turner syndrome. Osteoporos Int. 2010;21:2093-2099.

10. Pluskiewicz W, Łuszczyńska A, Halaba Z, Drozdzowska B, SońtaJakimczyk D. Skeletal status in survivors of childhood acute lymphoblastic leukemia assessed by quantitative ultrasound: A pilot cross-sectional study. Ultrasound Med Biol. 2002;28:1279-1284.

11. Pluskiewicz W, Adamczyk P, Drozdzowska B, Pyrkosz A, Halaba Z. Quantitative ultrasound and peripheral bone densitometry in patients with genetic disorders. Ultrasound Med Biol. 2006;32:523-528.

12. Halaba ZP. The usefulness of phalangeal quantitative ultrasound in the assessment of skeletal status in children and adolescents. Curr Med Imaging Rev. 2008;4:194-201.

13. Drozdzowska B, Pluskiewicz W, Halaba Z, Misiołek H, Beck B. Quantitative ultrasound at the hand phalanges in 2850 females aged 7 to 77 yr: A cross-sectional study. J Clin Densitom. 2005;8(2):216-221.

14. Halaba ZP, Pluskiewicz W. Quantitative ultrasound in the assessment of skeletal status in children and adolescents. Ultrasound Med Biol. 2004;30(2):239-243.

15. Eunice Kennedy Shriver National Institute of Child Health and Human Development: Bone mineral density in childhood study. https:// bmdcs.nichd.nih.gov/zscore.htm Accessed December 14, 2015 April 10, 2016.

16. Zemel BS, Leonard MB, Kelly A, et al. Height adjustment in assessing dual energy $\mathrm{X}$-ray absorptiometry measurements of bone mass and density in children. J Clin Endocrinol Metab. 2010;95:1265-1273.

17. Landin LA. Fracture patterns in children. Analysis of 8,682 fractures with special reference to incidence, etiology and secular changes in a Swedish urban population 1950-1979. Acta Orthop Scand Suppl. 1983;202:1-109.

18. Gravholt CH, Lauridsen AL, Brixen K, Mosekilde L, Heickendorff, Christiansen JS. Marked disproportionality in bone size and mineral, and distinct abnormalities in bone markers and calcitropic hormones in adult Turner syndrome: A cross-sectional study. J Clin Endocrinol Metab. 2002;87:2798-2808.

19. Bakalov V, Chen M, Baron J, et al. Bone mineral density and fractures in Turner syndrome. Am J Med. 2003;115(4):259-264.

20. Hansen S, Brixen K, Gravholt CH. Compromised trabecular microarchitecture and lower finite element estimates of radius and tibia bone strength in adults with turner syndrome: A cross-sectional study using high-resolution-pQCT. Bone Miner Res. 2012;27(8):1794-1803.
21. Nour MA, Burt LA, Perry RJ, Stephure DK, Hanley DA, Boyd SK. Impact of growth hormone on adult bone quality in Turner syndrome: A HRpQCT study. Calcif Tissue Int. 2016;98(1):49-59.

22. Bechtold S, Rauch F, Noelle V, et al. Musculoskeletal analyses of the forearm in young women with Turner syndrome: A study using peripheral quantitative computed tomography. J Clin Endocrinol Metab. 2001;86:5819-5823.

23. Bakalov VK, Axelrod L, Baron J, et al. Selective reduction in cortical bone mineral density in Turner syndrome independent of ovarian hormone deficiency. J Clin Endocrinol Metab. 2003;88:5717-5722.

24. Zuckerman-Levin N, Yaniv I, Schwartz T, Guttmann H, Hochberg Z. Normal DXA bone mineral density but frail cortical bone in Turner's syndrome. Clin Endocrinol. 2007;67:60-64.

25. Neely EK, Marcus R, Rosenfeld RG, Bachrach L. Turner syndrome adolescents receiving growth hormone are not osteopenic. J Clin Endocrinol Metab. 1993;76(4):861-866.

26. Vierucci F, Del Pistoia M, Erba P, Federico G, Saggese G. Usefulness of phalangeal quantitative ultrasound in identifying reduced bone mineral status and increased fracture risk in adolescents with Turner syndrome. Hormones. 2014;13(3):353-360.

27. Soucek O, Schonau E, Lebl J, Sumnik Z. Artificially low cortical bone mineral density in Turner syndrome is due to the partial volume effect. Osteoporos Int. 2015;26(3):1213-1218.

28. Bakalov V, Bondy CA. Fracture risk and bone mineral density in Turner syndrome. Rev Endocr Metab Disord. 2008;9(2):145-151.

29. Soucek O, Lebl J, Snajderova M, et al. Bone geometry and volumetric bone mineral density in girls with Turner syndrome of different pubertal stages. Clin Endocrinol (Oxf). 2011;74:445-452.

30. Cooper C, Dennison EM, Leufkens HG, Bishop N, van Staa TP. Epidemiology of Childhood Fractures in Britain: A study using the general practice research database. J Bone Miner Res. 2004;19:1976-1981.

31. Konstantynowicz J, Bialokoz-Kalinowska I, Motkowski R, et al. The characteristics of fractures in Polish adolescents aged 16-20 years. Osteoporos Int. 2006;16(11):1397-1403.

32. Schönau E, Radermacher A, Wentzlik U, Klein K, Michalk D. The determination of ultrasound velocity in the os calcis, thumb and patella during childhood. Eur J Pediatr. 1994;153:252-256.

33. Baroncelli Gl, Federico G, Bertelloni S, et al. Bone quality assessement by quantitative ultrasound of proximal phalanxes of the hand in healthy subjects aged 3-21 years. Pediatr Res. 2001;49(5):713-718.

34. Nadeem M, Roche EF. Bone mineral density in Turner's syndrome and the influence of pubertal development. Acta Paediatr. 2014;103(1): e38-42.

35. Ari M, Bakalov VK, Hill S, Bondy CA. The effects of growth hormone treatment on bone mineral density and body composition in girls with turner syndrome. J Clin Endocrinol Metab. 2006;91:4302-4305.

36. Högler W, Briody J, Moore B, Garnett S, Lu PW, Cowell CT. Importance of estrogen on bone health in Turner syndrome: A cross-sectional and longitudinal study using dual-energy X-ray absorptiometry. J Clin Endocrinol Metab. 2004;89:193-199. 